\title{
Importancia del turismo en la promoción de políticas públicas en favor del desarrollo
}

\section{regional sostenible}

The importance of tourism in promoting public policies in support of sustainable regional development

Importância do turismo na promoção de políticas públicas em prol do desenvolvimento sustentável regional

\section{Resumen}

El desarrollo regional del turismo en Brasil se presenta como una importante alternativa para impulsar la implementación de políticas públicas dirigidas a la inclusión social y a la superación de las diferentes barreras y desafíos para tornar esta actividad factible según criterios de sostenibilidad ambiental. En lo que respecta a la política de desarrollo regional desde la perspectiva de la sustentabilidad, el turismo debe estructurarse bajo tres ejes básicos: i) económico; ii) social y iii) ambiental. Ante este marco, el objetivo de este artículo consiste en analizar el "Programa de Regionalización del Turismo" en Brasil con miras a hacer posible diseñar una visión global de su papel como herramienta positiva para el desarrollo socioeconómico del turismo brasileño. Para alcanzar dicho objetivo se retoma el punto de vista de que el turismo es una actividad que permite minimizar los impactos económicos, ambientales y sociales negativos que se derivan de su inserción en ecosistemas naturales y comunidades, generando beneficios para la sociedad. La metodología utiliza herramientas básicas de análisis documental para clasificar la información del Programa de Regionalización del Turismo, parte integrante del Plan Nacional de Turismo de Brasil. Los resultados obtenidos sirven de base para apuntar al turismo como una actividad atractiva y con gran potencial para desarrollar regiones brasileñas, aunque se exige que sean consideradas las especificidades de las ciudades y poblaciones. Se pudo observar aun que, en lo que se refiere a la sostenibilidad, debe ser dada especial alusión también a los aspectos sociales, culturales y ambientales de modo a tornar el proceso turístico un vehículo para fomentar el crecimiento sostenible, a través del logro de los imperativos del desarrollo económico, al tiempo que minimiza los impactos sociales, culturales y ambientales negativos.

Palabras clave: Desarrollo sostenible; Políticas Públicas; Desarrollo local; Política social; Turismo regional.

\begin{abstract}
Regional development of tourism in Brazil is presented as an important alternative to promote the implementation of public policies aimed at social inclusion and overcoming of the different barriers and challenges so as to render tourism that is feasible according to the Environmental Sustainability Principles. Viewing from the perspective of sustainability within the policy of regional development, tourism should be structured under three basic dimensions: i) economic; ii) social and iii) environmental. Based on this framework, the aim of this paper is to analyze the "Program of Regionalization of Tourism" in Brazil in order to make possible the design of its global role a as a positive tool for the socioeconomic development of Brazilian tourism. For this purpose, the study presumes that tourism as an activity can minimize the negative economic, environmental and social impacts which result from its insertion in natural ecosystems and communities, generating benefits for society. We use basic tools of document analysis to interpret the
\end{abstract}

\footnotetext{
${ }^{1}$ Alumna del Programa de Doctorado en Ingeniería de Producción y Sistemas (CEFET/RJ - PPPRO). Magíster en Administración por la Universidad Federal Fluminense (PPGAdUFF). Licenciada en Contabilidad por Universidad Estatal de Piauí (UESPI) y en Administración por la Facultad de Actividades Empresariales de Teresina (FAETE).

${ }^{2}$ Profesor Catedrático en el Programa de Postgrado en Administración de la Facultad de Administración y Contabilidad de la Universidad Federal Fluminense (PPGAD - UFF). Doctor en Contabilidad y Finanzas por la Facultad de Economía y Empresas (Universidad de Zaragoza, España). Magíster en Contabilidad por la Universidad del Estado de Río de Janeiro (UERJ). Licenciado en Administración por la Universidad Federal Fluminense (UFF) y en Contabilidad por la Universidad del Estado de Río de Janeiro (UERJ). Académico Titular de la Cátedra no 199 de la Academia Nacional de Economía (ANE, Brasil).
} 
data from Tourism Regionalization Program, an integral part of the Brazilian National Tourism Plan. The results serve as a basis for targeting tourism as an attractive activity with considerable potential to develop Brazilian regions, although demanding that the peculiarities of cities and populations be considered. It was observed that, as to sustainability, special attention should also be given to social, cultural, and environmental aspects in order to turn the tourism process into a vehicle able to promote sustainable growth, through the achievement of the imperatives of economic development, while minimizing negative social, cultural, and environmental impacts.

Keywords: Sustainable development; Public Policies; Local development; Social policy; Regional tourism.

\section{Resumo}

O desenvolvimento regional do turismo no Brasil apresenta-se como uma importante alternativa para promover a implementação de políticas públicas voltadas para a inclusão social e a superação das diversas barreiras e desafios para viabilizar essa atividade alinhada a critérios de sustentabilidade ambiental. No que se refere à política de desenvolvimento regional numa perspectiva de sustentabilidade, o turismo deve se estruturar em três eixos básicos: i) econômico; ii) social e iii) ambiental. Diante deste contexto, o objetivo deste artigo é analisar o "Programa de Regionalização do Turismo" no Brasil, a fim de traçar uma visão global de seu papel como instrumento positivo para o desenvolvimento socioeconômico do turismo brasileiro. Para tal, este estudo parte do pressuposto de que o turismo como atividade pode minimizar os impactos econômicos, ambientais e sociais negativos decorrentes de sua inserção em ecossistemas naturais e em comunidades, gerando benefícios para a sociedade. A metodologia utiliza ferramentas básicas de análise documental para classificar as informações do Programa de Regionalização do Turismo, parte integrante do Plano Nacional de Turismo do Brasil. Os resultados obtidos servem de base para apontar o turismo como uma atividade atrativa, com grande potencial de desenvolvimento das regiões brasileiras, embora seja necessário levar em consideração as especificidades das cidades. Além disso, observou-se que, no que se refere à sustentabilidade, atenção especial também deve ser dada aos aspectos sociais, culturais e ambientais para tornar o processo turístico um veículo de promoção do crescimento sustentável, por meio do cumprimento dos imperativos do desenvolvimento econômico, minimizando os negativos impactos sociais, culturais e ambientais resultantes dessa atividade.

Palavras-chave: Desenvolvimento sustentável; Políticas Públicas; Desenvolvimento local; Política social; Turismo regional.

\section{Introducción}

Aunque el turismo sea asumido como una actividad económica alternativa (González, 2007, p. 54), los cambios que se han producido en la estructura económica mundial han convertido el turismo en uno de los principales sectores del comercio internacional y uno de los más importantes creadores de riqueza y motor clave para el progreso socioeconómico en las naciones de todo el mundo (UNWTO, 2013, p. 8). Hoy, el turismo moderno significa empleos, erradicación de la pobreza, igualdad de género y la protección y promoción del patrimonio natural y cultural de la humanidad.

De ahí que el turismo como actividad comercial estructurada se configura como un importante segmento de mercado que impulsa la economía mundial en la generación de empleo, renta y desarrollo económico. Según el Secretario General de la Organización Mundial del Turismo, Zurab Pololikashvili, el sector de turismo es mucho más de lo que se imagina, convirtiéndose en una "verdadera locomotora mundial de crecimiento económico y desarrollo, que impulsa la creación de más y mejores puestos de trabajo y sirve de catalizador de la innovación y el emprendimiento, ayudando a mejorar la vida de millones de personas y a transformar comunidades enteras" (OMT, 2019, p. 2). Además, es un sector de actividad que ha sido menos impactado por las crisis financieras que han asolado la economía mundial, aunque esta última crisis provocada más recientemente por la pandemia del "Corona Virus Disease y 2019", popularmente denominado COVID-19, esté causando grandes implicaciones para el desarrollo de dicha actividad tanto en niveles nacionales como internacional. Aun así, según la UNWTO (OMT, 2020, p. 2-3), el turismo ha experimentado una expansión continua a lo largo de los años, a pesar de algunos choques ocasionales, lo que demuestra la fortaleza del sector.

A pesar del fuerte impacto que ha tenido la pandemia en el Mundo, y por consecuencia la desaceleración económica mundial, el turismo internacional sigue mitigando el impacto socioeconómico y acelerando su proceso de recuperación, manteniendo una línea ascendente por el décimo año consecutivo de crecimiento continuo, alcanzando los 1,5 billones de USD de ingresos y un total de llegadas internacionales alrededor de 1.460 millones de personas en 2019 (equivalentes a un crecimiento real frente 
a 2018 del 4\% y 3\%, respectivamente). Para eso han contribuido la recuperación de destinos que habían sido afectados por temas de seguridad en los últimos años, el sólido restablecimiento de la demanda en los mercados emisores emergentes de Brasil y la Federación de Rusia tras algunos años de declive, además de una economía global relativamente fuerte, con un crecimiento de las clases medias y una rápida urbanización en las economías emergentes, así como por viajes más asequibles, una mayor facilitación de los viajes, avances tecnológicos y nuevos modelos de negocio (OMT, 2018, p. 4; OMT, 2020, p. 6).

No obstante, con su importante contribución a la economía el turismo presenta impactos negativos que necesitan ser controlados de modo a poder atender a las demandas de la sociedad y del medio ambiente. En parte porque el modelo lineal económico imperante hoy día no es más sostenible (Steffen et al., 2015), ya que está alcanzando sus límites físicos al «tomar, hacer, usar, tirar» confiando en la disposición de recursos fácilmente accesibles (Cerdá \& Khalilova, 2016, p. 11). Además, dicho "modelo económico mundial ha generado en la actualidad diversas y profundas transformaciones económicas, políticas, socioculturales y físicas que propician desequilibrios en las poblaciones rurales y urbanas por la falta de oportunidades para el mejoramiento en sus condiciones de vida" (Cruz \& Zizumbo, 2017, p. 945).

Como respuesta se empieza a delinear estrategias dirigidas hacia el turismo sostenible, en línea con el concepto de desarrollo sostenible, en aras de dar solución eficaz a los problemas de la actividad turística siguiendo un modelo turísticamente responsable; es decir, en palabras de Cruz y Zizumbo (2017, p. 945), introducir directrices públicas “de desarrollo nacional a partir de políticas, ordenamientos, programas, proyectos y acciones sociales que pretenden dar solución a las problemáticas a través de la intervención directa del Estado e incluso mediante la participación civil en la conformación de políticas sociales".

Estas circunstancias corresponden a un escenario dinámico donde el turismo se presenta como una actividad capaz de proporcionar el desarrollo de actividades productivas variadas abarcando prioritariamente las cuestiones ambientales y sociales. Ese enfoque redundará en un papel sostenible de las actividades turísticas, minimizando sus impactos económicos, ambientales y sociales negativos, además de generar beneficios económicos al promover el bienestar de la población local y proporcionar la conservación del patrimonio natural y cultural de las comunidades o regiones donde el turismo se desarrolla.

De ahí que la práctica de la actividad turística de forma no sostenible ya no atiende más a los anhelos de la sociedad, en vista de no tener como directriz atender a las necesidades de los turistas y las demandas socioeconómicas de los destinos receptores, considerando sus peculiaridades y especificidades. Por eso que la inclusión social del turismo como actividad económica viene ganando relevancia y dimensión en el contexto de las políticas públicas, dada su esencialidad y potencialidad para promover acciones que alcancen un nivel económico, social, cultural y ambiental favorable al desarrollo sostenible y a la sustentabilidad. Con este fin, el desarrollo del turismo regional se presenta como una de las principales alternativas para el desarrollo de políticas públicas dirigidas a la inclusión sostenible.

En este sentido, en el caso de Brasil, el desarrollo del turismo orientado a la sostenibilidad se basa en el Programa de Regionalización del Turismo (PRT), una política pública constituida a partir de los lineamientos del Plan Nacional de Turismo 2018-2022 brasileño "Más empleo e ingresos para Brasil", con el fin de promover la descentralización de decisiones y acciones tanto en el ámbito local (municipal), como en los niveles estatal y regional, fortaleciendo la integración entre gobierno, empresas, organizaciones sociales y comunidades locales en el desarrollo turístico de las regiones (Brasil, 2019b).

Frente a este contexto, la cuestión de investigación que se presenta es dirigida a "trazar un panorama del desarrollo regional del turismo en Brasil, diagnosticando a los huecos, iniciativas y éxitos basados en los objetivos y lineamientos establecidos en el PRT”, de modo a sentar las bases que sirvan como referencia para identificar: ¿cómo está la implementación del PRT brasileño?; ¿qué beneficios ha generado y cuáles son sus desafíos para la gestión y el desarrollo sostenible como actividad en las comunidades brasileñas?; y, finalmente, ¿qué limitaciones enfrenta como política pública dirigida al impulso del desarrollo local, tanto económico, como social y económico? 
Para dar respuesta a estos interrogantes, el objetivo de este artículo consiste en analizar el "Programa de Regionalización del Turismo" en Brasil con miras a hacer posible diseñar una visión global de su papel como herramienta positiva para el desarrollo socioeconómico del turismo brasileño. De hecho, será posible diseñar una visión global del papel del turismo como herramienta positiva para el desarrollo socioeconómico en Brasil, analizando y describiendo la política turística implementada en Brasil como un canal potencial del desarrollo regional y del crecimiento sostenible.

Frente a todo lo expuesto y basado en la investigación realizada, la estructura del artículo se compone de cinco apartados, siendo el primero esta introducción, donde se presentan el contexto y el problema estudiado, además del objetivo del artículo. En el segundo apartado se analiza el marco teórico de modo a: i) clarificar los factores críticos para caracterizar el turismo sostenible; ii) presentar las bases para la implementación de políticas públicas de turismo regionalizado; y iii) describir a los componentes regulatorios relacionados al programa de regionalización turística aprobado por el MTur en el año 2013. El tercer apartado presenta la metodología utilizada en el estudio, señalando los aspectos metodológicos y los procedimientos de investigación aplicados al estudio y su delimitación. El siguiente apartado contiene los datos recogidos y correspondientes análisis, relatando los resultados alcanzados con la investigación. A modo de cierre, se integra un último apartado donde se presentan las conclusiones y reflexiones finales sobre el tema, seguidas de las referencias utilizadas en el análisis.

\section{Marco conceptual}

\subsection{Factores caracterizadores del turismo sostenible}

El sector turístico ejerce una importante contribución en el desarrollo socioeconómico dada su capacidad de generación de empleo y renta en diferentes servicios (Sansolo \& Cruz, 2003), lo que hace con que éste sea capaz de suplir la demanda por empleo de una población que vive al margen del modelo económico capitalista excluyente.

Según Ignarra (1999), la dinámica del turismo engloba los elementos que constituyen la oferta turística, divididos en: i) recursos naturales (clima, suelo, paisajes, fauna, flora y otros) y culturales (patrimonio arquitectónico, cultural local, gastronomía, artesanía y otros); ii) bienes y servicios (productos alimenticios, materiales deportivos, servicios receptivos, atracciones, etc.); iii) infraestructura y equipamientos (carreteras, hoteles, posadas, restaurantes, etc.); iv) gestión (la forma en que el producto se gestiona y se ofrece); v) la imagen de la marca (como el producto es percibido por los consumidores); y vi ) el precio (el valor que hay que pagar debe ajustarse a los beneficios ofrecidos).

Además, el producto turístico debe considerar aún a los elementos que integran el destino turístico: i) los recursos disponibles; ii) la cultura de la población; y iii) la vocación turística. El análisis de todos estos aspectos es fundamental para que se ofrezca un producto acorde con las características locales y competitivas para el mercado (CNM, 2012).

Se sabe que la cuestión regional brasileña ya ha sido analizada, en las últimas décadas, de manera muy amplia, a la luz de diferentes contextos socioeconómicos e institucionales, así como bajo la influencia de diferentes armazones metodológicos. De esta forma, estudios académicos y evaluaciones de agencias gubernamentales sobre este tema tan relevante para dicho país han sido orientadores e influyentes al debate en torno al contexto de las políticas regionales (Monteiro Neto, Castro \& Brandão, 2017). Sin la pretensión de discutir sobre las implicaciones y principales legados de dichas políticas, se toma como premisa que la actividad turística, de una forma general, es importante para la generación de empleo y la elevación del nivel de renta de una comunidad habitante de determinada región y, para eso, hay que ser ejecutado de forma sostenible.

En el contexto del turismo y su enfoque hacia la sostenibilidad, según la OIT (2011, p. 13), esta actividad tiene como compromiso "aumentar el progreso de la localidad, mediante la maximización de la contribución del turismo a la prosperidad económica del lugar de destino". Bajo ese enfoque, el principal objetivo del turismo es "generar ingresos y trabajo decente para los trabajadores, sin afectar el entorno y la cultura del lugar de destino de los turistas, y garantizar la viabilidad y 
competitividad de los destinos y las empresas, para que puedan continuar prosperando y generando beneficios a largo plazo".

El turismo desde la perspectiva de la sustentabilidad está compuesto por tres pilares: la justicia social, el desarrollo económico, y la integración del medio ambiente. En este sentido, el desarrollo económico debe ser una experiencia positiva para las poblaciones locales, las empresas de turismo, los trabajadores y los propios turistas. Además, teniendo en cuenta que las perspectivas de desarrollo sostenible se articulan desde diversos enfoques, entre ellos el "triple bottom line": económico, social y ambiental, "el turismo sostenible debe utilizar de la mejor forma posible los recursos ambientales que constituyen el elemento fundamental del desarrollo turístico, manteniendo los procesos ecológicos esenciales, y ayudando a conservar los recursos naturales y la biodiversidad" (OIT, 2011, p. 17). Frente a tales perspectivas, el turismo sostenible se articula aún en acciones que buscan lograr la rentabilidad de los negocios, considerando la satisfacción de los clientes y, más aún, pautado en el trabajo decente en los destinos turísticos receptores de dicha actividad. La Figura 1 muestra un diagrama para lograr simultáneamente el turismo y el desarrollo sostenible con el objeto de reducir la pobreza, mejorar la vida de las personas y conservar los recursos naturales en un mundo.

Figura 1. Componentes del desarrollo sostenible.

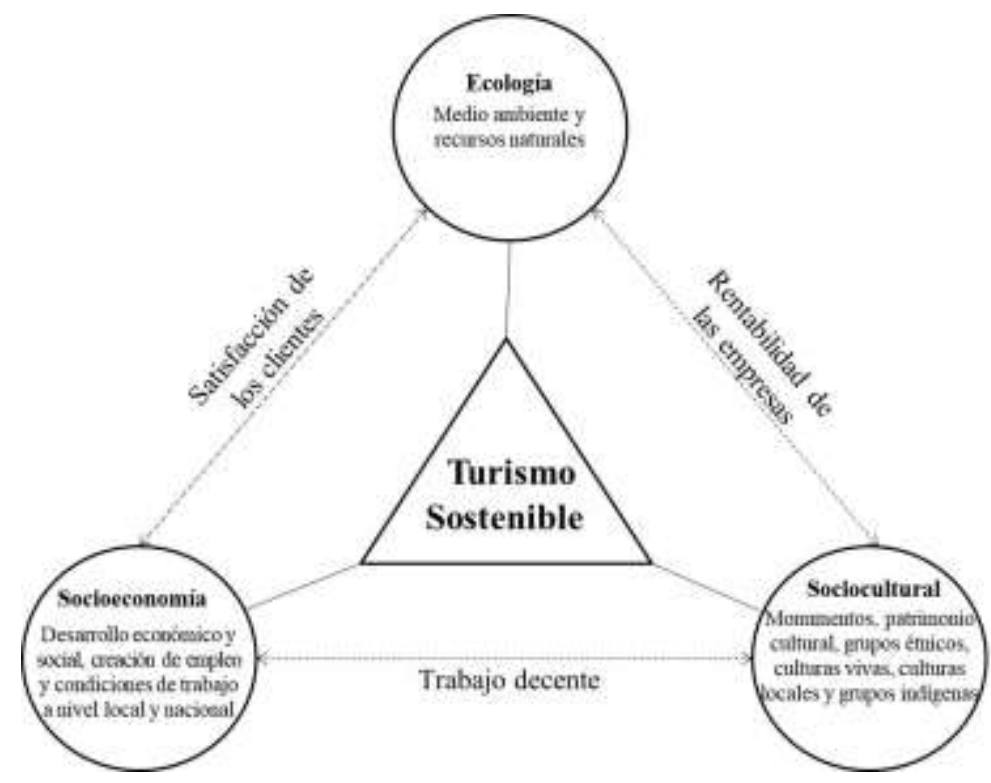

Fuente: Adaptado de OIT (2010, p. 53).

Como un propulsor del desarrollo local, el turismo sostenible debe fortalecer el comercio de servicios y crear empleos (UNWTO, 2013). Sin embargo, debe, también, considerar los aspectos relacionados al contexto de su actuación. Es decir, una política de turismo sostenible debe "respetar la autenticidad sociocultural de las comunidades anfitrionas, conservar su reconocido patrimonio cultural vigente y sus valores tradicionales, y contribuir al entendimiento y la tolerancia interculturales; a la vez que asegurar actividades económicas viables a largo plazo, que proporcionen beneficios socioeconómicos equitativos para todas las partes interesadas, incluyendo el empleo estable, las oportunidades de obtener ingresos y servicios sociales para las comunidades receptoras, y que contribuyan al alivio de la pobreza" (OIT, 2011, p. 17).

Para quedarse en línea con dicha perspectiva, las estrategias para la formulación del turismo orientado hacia formas de desarrollo sostenible deben basarse en la asimilación del espacio preservando los niveles de calidad ambiental e integrando los niveles de bienestar social y laboral de las comunidades receptoras (Gonzáles, 2006, p. 362-363). 


\subsection{Papel del turismo en el desarrollo regional}

Debido a su papel como actividad económica, el turismo ocupa posición destacada en la promoción de forma integrada del desarrollo sostenible en las regiones donde viven las poblaciones con perspectivas de calidad de vida más baja y con poca s inversiones sociales.

Sin embargo, históricamente, las inversiones en el sector de turismo son dirigidas a destinos turísticos que tradicionalmente ofrecen facilidades al ejercicio del turismo, en especial en términos como atractivos (sol y playa), infraestructura (hotelería, gastronomía y transporte) o equipamientos turísticos y de apoyo (accesibilidad). De esa forma, regiones más lejanas o inhóspitas geo-morfo-climáticas que no gozan de buenas vías de acceso y mano de obra especializada, en general, quedan ignoradas. Con eso, se acaba dejando de "promover el desarrollo del turismo como agente de transformación social, fuente de riqueza económica y desarrollo social” (Brasil, 2007, p. 43).

Para Cota (2011, p. 139), toca al Estado crear estrategias sociales dirigidas a satisfacer las necesidades de la población mediante un conjunto de intervenciones que permitan "corregir o compensar la desigualdad que genera el libre juego de la fuerza del mercado". Eso implica una verdadera transformación del modelo económico dominante de modo a conjugarse un nuevo contexto de interacciones donde se crean condiciones para promover un mundo más equitativo, asegurando "el progreso en el medio ambiente, la disminución de la desigualdad social, la erradicación de la pobreza extrema" y aminorando la inseguridad pública y la marginalización social (Artega \& Solis, 2001, p. 14-16).

En base a eso, la actividad turística debe ser practicada, en su perspectiva económica, como una alternativa para el desarrollo de las localidades con bajo índice de desarrollo económico pero poseedoras de potencial turístico. De ahí que, “en las últimas décadas varias comunidades han buscado mejorar sus condiciones mediante su incorporación en proyectos turísticos"; no obstante, "no todas han logrado consolidar los proyectos e incentivar el desarrollo local" (Cruz \& Zizumbo, 2017, p. 944). Es decir, tal como ocurre actualmente, "las políticas públicas dirigidas a la actividad turística han dejado al margen a la población de los destinos trabajados y no han generado los beneficios económicos para las comunidades locales, configurándose en una actividad no sustentable con ausencia de políticas públicas eficaces” (Silva \& Andrade, 2008, p. 15).

Si bien planteadas e implementadas, las políticas públicas pueden asumir un papel protagónico como impulsor de la economía regional, incentivando el desarrollo local y generando beneficios sociales a una parte carente de la población, además de propiciar contribución para aminorar los desequilibrios sociales locales e impulsar el bienestar social. Por lo tanto, para lograr el mejoramiento en las condiciones de vida en las poblaciones desprotegidas socialmente, es papel fundamental del Estado, juntamente con otros actores económicos, aprovechar los recursos locales incorporados a las actividades turísticas, de modo a promover la búsqueda de soluciones para resolver los problemas que enfrentan dichas comunidades, especialmente en aquellas regiones en situación de pobreza.

Para emprender esa embestida transformadora, no obstante, hay que cambiar la manera como se organiza el turismo en las localidades, ya que esta actividad es vendida como un producto y, no raro, acaba marginalizando a las comunidades que pertenecen a esas regiones. En consecuencia, la gran mayoría de la población queda fuera de las discusiones sobre el desarrollo del turismo en la localidad que teóricamente les pertenece. Para Silva \& Andrade (2008, p. 17), “el desarrollo económico local es una estrategia de desarrollo por la cual la comunidad asume nuevo papel: el de comunidad demandante, del que emerge como agente, protagonista y emprendedor, con autonomía e independencia”.

Dicho de otra forma, en palabras de Vargas (2018, p. 640), “como sujeto de desarrollo, la región constituye una instancia necesaria para construir el andamiaje donde la acción de la política pública de los actores reconocidos, accionan los procesos de gestión institucional para construir un proyecto regional que permita generar riqueza y crear beneficios que se direccionen al logro de la equidad social”. Dentro de toda esa dinámica, la actividad turística posee un claro potencial para promover el desarrollo regional; pero para que el turismo asuma papel preponderante en la generación de efectos iniciales en el 
desarrollo social, la atracción turística debe de funcionar como actividad motora sobre la región propiciando las bases para la complementariedad con las actividades locales en términos de difusión de las relaciones de compra y venta entre los agente presentes en la región y el retorno de la distribución de renta sobre las estructuras de consumo (Ablas, 1991, p. 50-52).

\subsection{Programa de Regionalización del Turismo brasileño}

El tema desarrollo regional en el contexto de políticas públicas ha ido ganando dimensión dada su esencialidad y posibilidades de transformaciones económicas y sociales, además de preservar el medio ambiente. Así, el desarrollo regional se caracteriza como una de las principales banderas de las políticas públicas en Brasil, en éstas se incluyen el desarrollo regional del turismo sostenible. Según el MTur (Brasil, 2013a), la actividad turística sostenible tiene como directriz satisfacer las necesidades de los visitantes y las necesidades socioeconómicas de las regiones receptoras, considerando sus peculiaridades y especificidades. El Plan Nacional de Turismo 2018-2022 (Brasil, 2018) tiene como propuesta estimular acciones que puedan atender a las necesidades de regiones menos desarrolladas de modo a promover la regionalización del turismo.

A partir de la promulgación de la Constitución de 1988 (Brasil, 1988), se retomó el proceso de desarrollo brasileño, a través de varias políticas públicas, entre las cuales está inclusa la política de fomento regional con el objetivo de reducir las desigualdades sociales regionales. En este contexto, surge la política nacional de turismo brasileña con enfoque territorial que comenzó con la institucionalización del Programa Nacional de Municipalización del Turismo - PNMT, en 1994, coordinada por el Ministerio de Industria, Comercio y Turismo. El principal objetivo de ese programa ha sido desarrollar el turismo en diferentes contextos productivos. Para el alcance de tal propósito, fue concebido el primero PNMT (Embratur, 1999; 2002) para dinamizar el desarrollo de la actividad turística en el ámbito municipal. Para el MTur (Brasil, 2013a, p. 17), el histórico y las evaluaciones de las políticas nacionales para el turismo en Brasil revelan que el PNMT fue más allá de un simple programa de gobierno, transformándose en un movimiento nacional capaz de movilizar agentes económicos y producir resultados sociales, que posibilitaron avanzar hacia el alcance territorial como estrategia para el fomento de las actividades del turismo en el país.

Creado en 2004, el PRT se constituye en una política pública, en ámbito territorial (Brasil, 2004a; 2004b), a partir del Plan Nacional del Turismo 2003-2007 - PNT (Brasil, 2003), que determinó como macro programa estructurante la "Estructuración y Diversificación de la Oferta Turística" (Brasil, 2013a, p. 17). En cuanto política pública, el turismo se constituye como importante mecanismo para que las acciones gubernamentales sean coherentes para alcanzar las metas establecidas (Vieira, 2011). En ese sentido, "la consecución de la política nacional de turismo de Brasil se centró en el propósito de que su ejecución de forma descentralizada y regionalizada, con foco en la planificación coordinada y participativa, repercutiese positivamente en los resultados socioeconómicos del territorio" (Brasil, 2018, p. 55).

Conforme el MTur (Brasil, 2013a, p. 17), “el programa se inició con la participación efectiva de los representantes de los órganos y colegios de turismo municipales y estatales, además de representantes de la iniciativa privada, de las instituciones de enseñanza y del tercer sector". A partir del debate, por medio de talleres y reuniones sectoriales y nacionales, fue posible reflexionar sobre los conceptos, principios y valores referentes a los grupos de representantes de los sectores de la cadena de turismo (público, privado y sociedad civil organizada) y definir las estrategias para implantación del programa en las regiones mapeadas (27 unidades federativas de Brasil). A continuación, el planeamiento de las acciones y, en particular, la construcción de criterios, a partir de un proceso plural y democrático, permitió definir las "Instancias de Gobernanza", constituyendo un modelo de formación e institucionalidad definido y constituido a partir de las realidades regionales y sus relaciones intermunicipales. El conjunto de municipios que constituirían la "región turística" para promover la sostenibilidad, la inclusión y la diversidad de cada una de las 219 regiones turísticas identificadas, en la época, agrupaban en su conjunto a 3.319 municipios. Ese proceso generó una herramienta de trabajo denominada "Mapa de la Regionalización del Turismo" (Brasil, 2014; 2016, 2019d), que tenía como objetivo orientar acciones de programas y procesos en el ámbito del Ministerio de Turismo. 
Para identificar el desempeño del sector se creó la categorización de los municipios, que es un documento para implementar el PRT, elaborado basado en una matriz diagnóstica construida de forma matricial con base en los ejes de actuación del programa de regionalización. Como herramienta estratégica de desarrollo de política pública, el PRT brasileño tiene el objetivo de subsidiar acciones que promuevan la estructuración y calificación de las regiones turísticas. A través de trabajo colectivo las acciones resultan en beneficios para los municipios involucrados, de esa forma el turismo se consolida como una actividad capaz de generar empleos e ingresos, reduciendo las desigualdades regionales y promoviendo la inclusión social y productiva de las regiones (Brasil, 2013a, p. 24).

Para la implementación del programa de regionalización se planificaron algunas etapas denominadas de módulos operativos, constituidas según los criterios que marcan el norte de las demandas de las actividades turísticas en las regiones y en los municipios, además de una secuencia de acciones estratégicas a ser implementadas buscando alcanzar los objetivos propuestos en el programa. Con eso, fueron trazados ocho ejes de actuación con enfoque territorial para el PRT, cuya propuesta define los mecanismos de orientación a las acciones de apoyo a la gestión, estructuración y promoción del turismo en las regiones y municipios (Brasil, 2013a). El método de regionalización del turismo propuesto en su reformulación definió las acciones estratégicas bajo la perspectiva de encaminar las demandas de las actividades turísticas en las regiones y municipios y, para alcanzar los resultados deseados, detalló las directrices que se constituyen en las premisas para su ejecución (Brasil, 2013a, p. 23).

Respecto a los ajustes provocar y promover concepciones innovadoras al enfrentamiento de las fragilidades del turismo brasileño, hay que definir la capacidad de la cadena productiva del turismo, además de comprender que la estructuración de los destinos turísticos depende de una nueva visión integradora del desarrollo productivo y de la competitividad, lo que exige la formalización de los servicios y la calificación de las personas. También en el campo empresarial hay que migrar hacia un modelo que provoque la ampliación de los micro y pequeños emprendimientos, y de las organizaciones asociativas y cooperativas, requiriendo la articulación en redes y demandando inversiones en tecnologías que busquen la oferta de productos y servicios segmentados y que agreguen valores del patrimonio sociocultural y ambiental y generen como resultado la ampliación de la capacidad de producción, de puestos de ocupación, de difusión y de distribución de productos y servicios, además de la circulación de la renta en el territorio.

Como se trata de una actividad que involucra muchos actores en Brasil, son necesarias políticas públicas y directrices orientadas a la implementación del desarrollo y el monitoreo del turismo brasileño alineado a diferentes proyectos y acciones activadas en diferentes ciudades en ese país (Dalonso, Lourenço, Remoaldo \& Panosso Netto, 2012, p. 186). De esta forma, la tarea de construir regiones turísticas también es competencia del gobierno, que en el ámbito nacional, instituyó la Coordinación Nacional del Programa a ser conducida por el MTur, a través del Comité Ejecutivo del Programa de Regionalización, constituyéndose en su núcleo de expresión técnico-político y actuando de forma coordinada con el Sistema Nacional de Turismo y la Cámara Temática de Regionalización del Consejo Nacional de Turismo. En el ámbito estadual, regional y municipal, las estructuras de la gestión pública y la cadena productiva del turismo, las instituciones de enseñanza superior y las organizaciones de la sociedad civil operan las acciones ejecutivas del PRT, apoyadas por los interlocutores actuando en los niveles estadual, regional y municipal. La Figura 2 presenta una visión panorámica del PRT brasileño según sus directrices política, estratégica y operativa de estructuración. 
Figura 2. Estructuración del PRT.
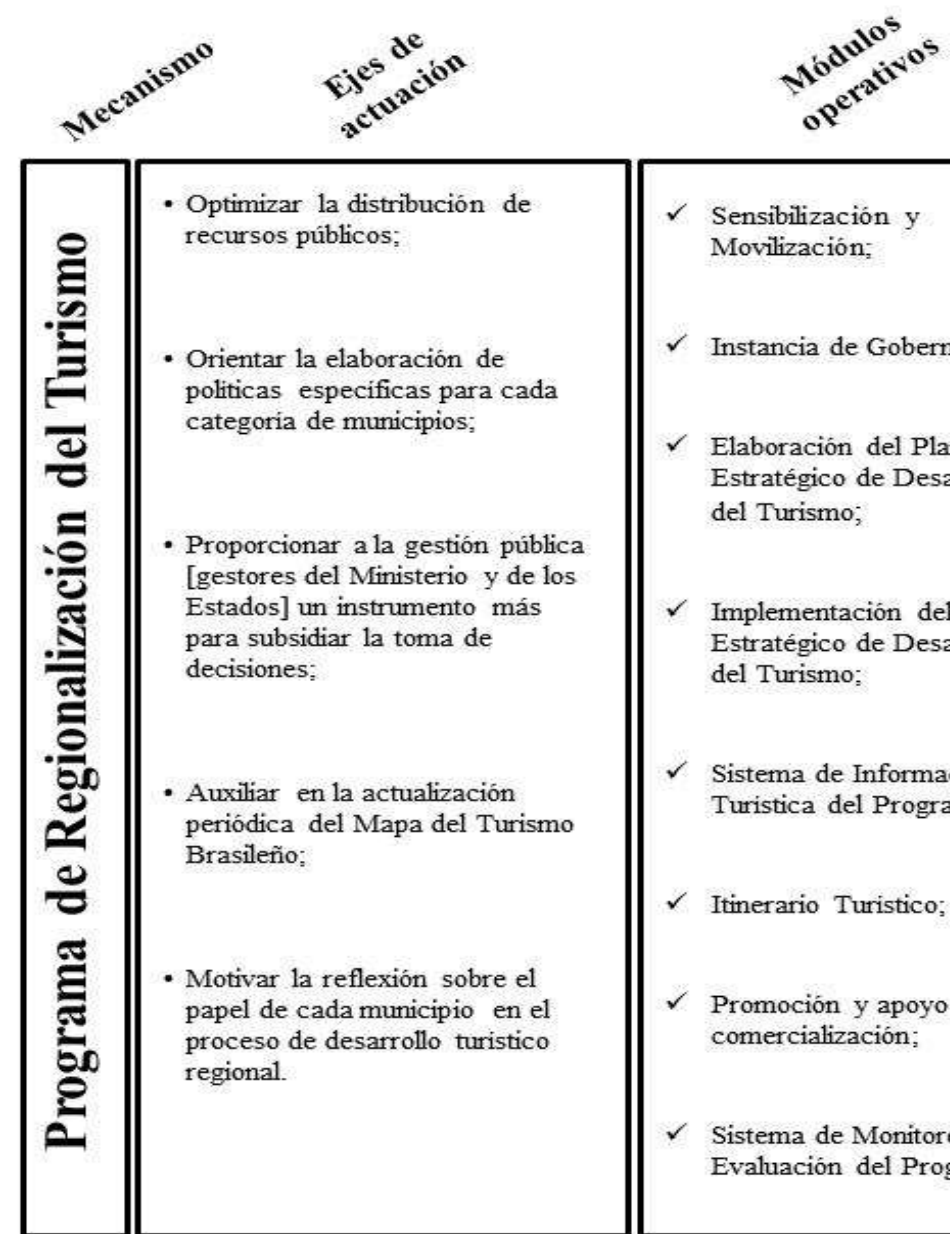

$\checkmark$ Sensibilización y Movilización:

$\checkmark$ Instancia de Gobernanza

$\checkmark$ Elaboración del Plan Estratégico de Desarrollo del Turismo;

$\checkmark$ Implementación del Plan Estratégico de Desarrollo del Turismo;

$\checkmark$ Sistema de Información Turistica del Programa;

$\checkmark$ Itinerario Turistico;

$\checkmark$ Promoción y apoyo a la comercialización;

$\checkmark$ Sistema de Monitoreo y Evaluación del Programa

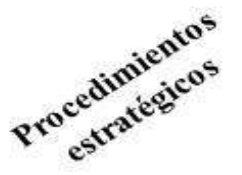

- Gestión descentralizada del Turismo;

- Planificación y Posicionamiento del Mesicionara

> Cualificación Profesional de los Servicios y de la

- Emprendedorismo, Captación y Promoción de Inversiones;

- Infraestructura Turistica:

Información al Turista;

Promoción y Apoyo a la Comercialización;

> Monitoreo.

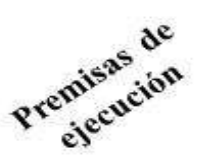

- Planificación regional: adoptar el enfoque del espacio regional como referencia para el desarrollo turistico.

- Integraciónsocial. buscar fortalecer la participación, por me dio de la generación de oportmidades de cmpleo y de espinturespent en el protagonismo de la cadena productiva del turisno en el ambito regional, en el conjunto de los municipios, y en los procesos de gestion de las politicas públicas.

- Inclusión social. entender la región como espacio plural y participativo, que amplia las capacidades humanas e institucionales, facilitando las relaciones politicas, económicas, sociales y culturales.

Descentralización: movilizar el abordaje territorial y el proceso de gestión descentralizada del turismo, actuando en el ambito del Sistema Nacional de Turismo, como forma de interactuar paraconsolidar las estrategias del Programa.

- Sostenibiliclact motivar el desarrollo sostenible de las regiones turisticas como base para la preservación de la identidad cultural, respetando las

- Innovación promover concepciones innovadoras al enfrentamiento de las fragilidades diagnosticadas, estimulando la cualificación profesional, de los servicios y de la producción asociada, integrando la diversidad de las expresiones culturales, agregando valor en todas las etapas de sus procesos
de producción y operación, y posibilitando la dinamización económica de las actividades en los territorios.

Competitividad: entender como la capacidad creciente de generar negocios en las actividades económicas relacionadas al sector del turismo, de forma competitividad del turismo brasileño.

Fuente: Elaborado basado en Brasil (2015b; 2013a; 2007; 2004a; 2004b) 
En este proceso de reestructuración, se rescata la representación del actor municipal como agente de desarrollo turístico, considerando ser él el poseedor de las realidades y demandas locales, se institucionaliza el representante regional, se garantiza la permanencia del interlocutor estadual como forma de fortalecer y extender la red de movilización (Brasil, 2013a: 25). Sin embargo, para el éxito de esta propuesta de programa como política turística de desarrollo local, es necesario que los agentes económicos (gobiernos, empresarios, sociedad civil, academia y tercer sector) actúen de forma integrada, sinérgica y armónica, en sintonía con las especificidades locales y los instrumentos operativos disponibles, tornando posible la calificación exigida para la gestión de la actividad turística.

En tal sentido, la regionalización encuentra puntos coincidentes con la política pública de turismo por traducir su mirada más allá del municipio, para fines de planeamiento, gestión, promoción y comercialización integrada y compartida de los destinos turísticos. Es decir, permite mirar la región y no más solo el municipio aisladamente (Brasil, 2019a).

Como parte del PRT, la regionalización se configura como un modelo de gestión descentralizada, otorgando a cada entidad federativa la interdependencia funcional para identificar alternativas de desarrollo según la densidad de flujos entre sus elementos, potencialidades, especificidades y realidades locales (Brasil, 2019b). De este modo, región y municipio articulan acciones de desarrollo turístico a través de los diversos actores sociales regionales involucrados en la actividad turística.

De acuerdo con las metas globales del Plan Nacional de Turismo 2018-2022 se pretende formular lineamientos que impulsen al sector turístico brasileño a través de la implementación del incremento de la entrada anual de visitantes internacionales de seis millones y quinientas mil personas para doce millones y de los ingresos generados de US\$ 6,5 mil millones para 19 mil millones de dólares. Respecto al número de viajes de turistas brasileños por todo el país, se desea ampliar de sesenta millones de personas para cien millones, además de expandir el número de empleos en el sector turístico de siete millones para nueve millones de puestos de trabajo. Sus directrices son: (i) construir y fortalecer regionalmente el turismo; (ii) mejorar la calidad y la competitividad en el sector turístico; y (iii) incentivar a la innovación (Brasil, 2019c)

Las metas y lineamientos apuntan al fortalecimiento del PRT a través del actual PNT, cuyo principal objetivo se centra en organizar las acciones del sector público en el desarrollo del turismo de manera integrada (Silva y Nogueira, 2020).

\section{Metodología}

La metodología de investigación utilizada se ha basado en análisis documental del Programa de Regionalización del Turismo (PRT), lo que impone al investigador un enfoque hermenéutico (Gonsalves, 2003, p. 68), siendo la interpretación del investigador fundamentalmente importante (Carvalho, Duarte, Menezes \& Souza, 2019). Además, tiene un carácter descriptivo y exploratorio (Ryan, Scapens, \& Theobald, 2002; Scapens, 2004; Adams, Hoque, \& McNichols, 2006) de modo a abordarse una realidad concreta (Berry \& Otley, 2004) que se sucede en el sector turístico brasileño.

Según Köche (2007, p. 124), en la investigación descriptiva no hay manipulación a priori de las variables, ya que la observación se hace a posteriori, verificando y evaluando las relaciones causales a medida que las variables se manifiestan espontáneamente en hechos, situaciones y condiciones que ya existen. Así, el enfoque descriptivo empleado ha posibilitado el análisis de las evidencias presentadas en la literatura, intentando buscar una aproximación al estado del arte relacionado a la temática turística y confrontarla con el Programa de Regionalización del Turismo y el Plan Nacional del Turismo, dos documentos centrales relacionados al desarrollo regional y al turismo sostenible en Brasil. Para eso se ha realizado el análisis documental (García, 1993; Bowen, 2009) buscando representar el contenido y la forma de dichos documentos y, así, describirlos y representarlos de forma unificada sistemática a partir de una estructura de datos que responda a la descripción general de los elementos que conforman tales documentos con las situaciones concretas observadas en la actividad turística en Brasil. Según Frey (2018), el análisis de documentos es una forma de investigación cualitativa que utiliza un procedimiento 
sistemático para analizar evidencia documental y responder preguntas de investigación específicas.

De ahí que el instrumento de recolección de datos utilizado se ha basado en la "técnica documental" (Pereira et al, 2018), teniendo en cuenta que las cuestiones abordadas son principalmente de naturaleza cualitativa y las informaciones analizadas se han generado principalmente a través de datos secundarios hechos disponibles por las organizaciones gubernamentales brasileñas. A continuación se hizo un análisis descriptivo documental (Pereira et al, 2018), basado en una comprensión profunda de los documentos divulgados por las autoridades ligadas al turismo en Brasil, buscando describir las características de una muestra y la relación entre los fenómenos, situaciones y eventos (Trípoli y Bender, 2010). Para esta labor se ha tomado como referencia, en primera instancia, un protocolo de investigación preliminar elaborado basado en la metodología propuesta por Massaro, Dumay y Guthrie (2016), donde se ha definido los criterios de inclusión y exclusión de los documentos recogidos en la base de datos, un marco analítico y la forma de codificación de los datos. Según Massaro, Dumay y Guthrie (2016), su protocolo ofrece menos sesgos y brinda más transparencia en los procedimientos de recolección de la investigación, además de proponer un mayor rigor en la revisión de la literatura frente a otras revisiones de la literatura tradicional, proporcionando la base para aumentar la confiabilidad de los hallazgos de la investigación.

\section{Resultados y Discusión}

El PRT en Brasil tiene como premisa fundamental compatibilizar el desarrollo turístico con la conservación y uso sostenible de los recursos existente en cada destino. Así, para perfeccionar la dinámica del turismo en las regiones turísticas brasileñas, priorizando las características comunes y fortaleciendo las interrelaciones entre los actores de esa actividad (sector público, sector privado y tercer sector), el Ministerio del Turismo de Brasil, en conjunto con el Instituto de Asesoría para el Desarrollo Humano - IADH, implementó un proceso de evaluación desarrollando una encuesta por medio de la aplicación de cuestionario en correo electrónico y la realización de talleres y entrevistas calificadas, donde han participado casi mil personas directamente comprometidas con el programa de regionalización del turismo brasileño en diversos niveles y unidades de la federación brasileña.

Basado en dicho material se puede hacer un diagnóstico concreto sobre los resultados generados por dicho programa y, también, analizar críticamente las necesidades futuras para alinear ese programa con acciones efectivas de desarrollo sostenible. La metodología adoptada por el MTur (Brasil, 2015a) permitió construir colectivamente las dimensiones, variables e indicadores que propician un proceso de autoevaluación basado principalmente en informaciones de naturaleza cualitativa que se vincula, directa o indirectamente, con la actividad de turismo en el ámbito regional.

Los datos base para la evaluación son estándar desde el enfoque de la "gestión del conocimiento". Por lo tanto, no se queda restricto solamente a la identificación de lo que ha funcionado o no, pero, por encima de todo, intenta rescatar las lecciones aprendidas y proponer alternativas de sustentabilidad a las acciones del programa en el futuro (Brasil, 2015a, p. 19).

En tal sentido se ha elaborado la "Matriz de Evaluación" (Brasil, 2015a, p. 27-41), construida en base a las proposiciones de los participantes del primer seminario y que han norteado todas las actividades y procedimientos realizados a continuación en dicha evaluación.

Respecto a los productos turísticos creados, la categorización es un instrumento elaborado por el MTur (Brasil, s.d.) para identificar el desempeño de la economía del sector turístico en el municipio. Este instrumento permite tomar decisiones más acertadas e implementar políticas que respeten a las peculiaridades de los municipios brasileños. Basado en dicha categorización de las regiones turísticas de los municipios se ha trazado el mapa del turismo brasileño, mostrado en la siguiente tabla: 
Tabla 1. Mapa del Turismo por Categorías (2013-2019).

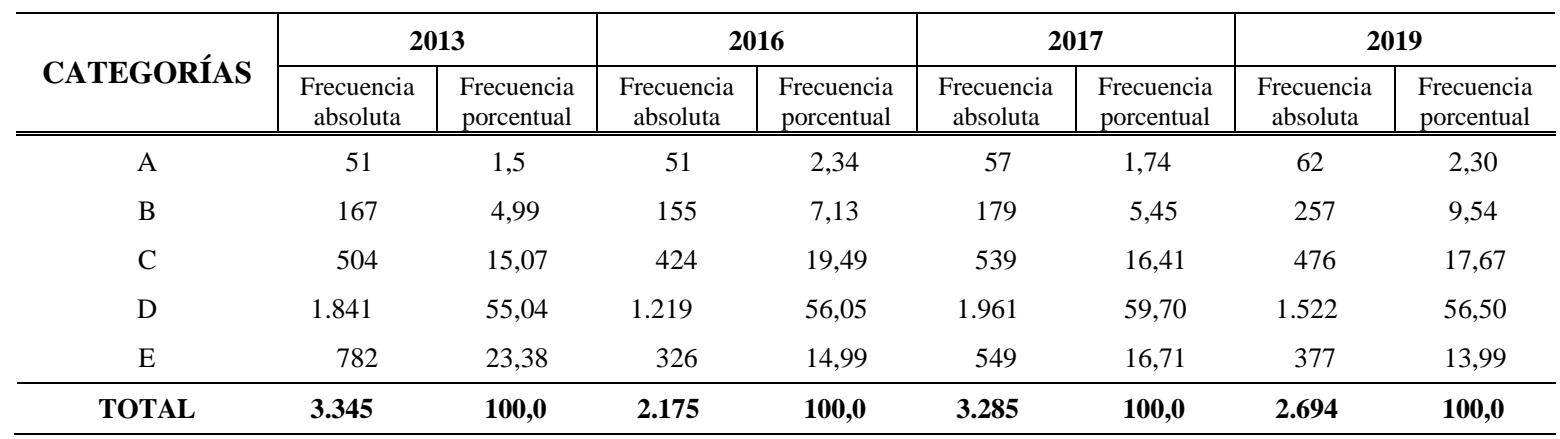

Fuente: Adaptado de MTur (s.d.).

En 2019, fueron analizados 5.570 municipios brasileños y, por esta razón, los valores promedio de las categorías cambiaron en la última actualización, lo que provocó que algunos municipios subieran o bajaran en la evaluación (Brasil, 2019a). Según el nuevo mapa, solo el dos por ciento de los municipios se ubican en la categoría A, que representa las ciudades con mayor flujo turístico y mayor creación de empleo y oferta de infraestructura hotelera. Se destaca, por lo tanto, que la actividad turística en los municipios incluidos en dicha categoría es considerada consolidada, contando incluso, con una demanda proporcionada por llegadas de turistas internacionales (Silva y otros, 2019). Sin embargo, cerca del 74,2\% de los municipios están clasificados en las categorías $\mathrm{C}$ y D, que representan, respectivamente, sitios donde la actividad turística aún está en estructuración y desarrollo o es incipiente, aunque contando con una demanda interna creciente. La categoría $\mathrm{E}$ abarca alrededor del 14\% de los municipios cuyo potencial turístico aún se encuentra en fase inicial de desarrollo. Según Silva y otros (2019, p. 9), estos municipios aún están involucrados en procesos burocráticos y de organización da infraestructura necesaria al turismo. Para los municipios de categoría B, casi un 10\%, cabe mencionar que existe ya el desarrollo de actividades turísticas, sin embargo, con mejor resultados del turismo nacional que de turistas internacionales.

El análisis comparativo del turismo entre las cinco regiones de Brasil, revela las diferencias macrorregionales en la oferta turística, conforme se resume en la Tabla 2 a continuación:

Tabla 2. Oferta turística por macrorregiones de turismo en Brasil.

\begin{tabular}{c|ccc|c|c|c|c|c|c}
\hline \multirow{2}{*}{ MACRORREGIONES } & \multicolumn{2}{|c|}{$\mathbf{2 0 1 3}$} & \multicolumn{2}{c|}{$\mathbf{2 0 1 6}$} & \multicolumn{2}{c|}{$\mathbf{2 0 1 7}$} & \multicolumn{2}{c}{$\mathbf{2 0 1 9}$} \\
\cline { 2 - 10 } & $\begin{array}{c}\text { Frecuencia } \\
\text { absoluta }\end{array}$ & $\begin{array}{c}\text { Frecuencia } \\
\text { porcentual }\end{array}$ & $\begin{array}{c}\text { Frecuencia } \\
\text { absoluta }\end{array}$ & $\begin{array}{c}\text { Frecuencia } \\
\text { porcentual }\end{array}$ & $\begin{array}{c}\text { Frecuencia } \\
\text { absoluta }\end{array}$ & $\begin{array}{c}\text { Frecuencia } \\
\text { porcentual }\end{array}$ & $\begin{array}{c}\text { Frecuencia } \\
\text { absoluta }\end{array}$ & $\begin{array}{c}\text { Frecuencia } \\
\text { porcentual }\end{array}$ \\
\hline \hline NORTE & 35 & 11,6 & 33 & 11,3 & 34 & 10,4 & 48 & 14,4 \\
NORESTE & 81 & 26,7 & 80 & 27,5 & 84 & 25,6 & 83 & 24,9 \\
CENTRO-OESTE & 36 & 11,9 & 35 & 12,0 & 36 & 11,0 & 33 & 9,9 \\
SUR & 49 & 16,2 & 53 & 18,2 & 53 & 16,2 & 54 & 16,2 \\
SUDESTE & 102 & 33,7 & 90 & 30,9 & 121 & 36,9 & 115 & 34,5 \\
\hline TOTAL & $\mathbf{3 0 3}$ & $\mathbf{1 0 0 , 0}$ & $\mathbf{2 9 1}$ & $\mathbf{1 0 0 , 0}$ & $\mathbf{3 2 8}$ & $\mathbf{1 0 0 , 0}$ & $\mathbf{3 3 3}$ & $\mathbf{1 0 0 , 0}$ \\
\hline
\end{tabular}

Fuente Adaptado de MTur (s.d.).

La categorización de 2019 abarcó a 2.694 municipios, constituyendo 333 regiones turísticas (Brasil, 2019a). El análisis de la distribución geográfica de la actividad turística según las macro-regiones de Brasil, revela el predominio de la región Sudeste en oferta de servicios turísticos, contando con un 34,5\% de la oferta, seguida de la región Noreste con un 25 por ciento. Las ciudades con desempeño más bajo están localizadas en el Centro-Oeste del país, seguido por el Norte, en vista del flujo turístico aún poco expresivo y sin la oferta de muchos empleos ni establecimientos turísticos. La búsqueda por estos sitios 
ocurre básicamente a partir de una demanda nacional [turistas brasileños] y su atractivo principal es referente a un producto turístico de características rural, ecoturismo y de aventura.

El programa trabaja desde la perspectiva de que incluso un municipio que no posee una clara vocación para el turismo, o sea que no recibe al turista en su territorio, puede beneficiarse de esta actividad si este municipio desempeña un papel de proveedor o proveedor de mano de obra, de trabajo o de productos destinados a atender al turista. Sin embargo, no obstante, la intencionalidad del PRT en promover la expansión del turismo en todo el país, existe un notorio desarrollo prioritario en los municipios costeros, con énfasis en las capitales y regiones metropolitanas, especialmente en las regiones Sudeste y Sur del país. Aunque el PRT contribuye efectivamente para definir las regiones y rutas turísticas, eran 200 regiones en 2006 y ya existen cerca de 333 según datos del 2019, Silva y Fonseca (2017, p. 150) son de opinión que los resultados de dicho programa son blandos en el sentido de interiorizar el turismo, teniendo en cuenta que sus objetivos iniciales no han sido alcanzados con eficiencia. Según dichos autores, los recursos financieros que se han ubicado en los municipios en las regiones turísticas se han hecho de manera desarticulada y ajena en relación a las definiciones y prioridades establecidas por el consejo de gestión de las regiones. Eso está generando dificultades a los agentes económicos y ha contribuido al vaciamiento del PRT, además de dificultar la expansión y consolidación del turismo en el interior del país.

En lo que le toca a la calidad de los productos turísticos creados, para el MTur (Brasil, 2005), al turismo hay que promoverlo como un producto turístico donde el conjunto de atractivos, equipamientos y servicios turísticos e instalaciones, ubicados en uno o más municipios, deben ser ofrecidos de forma organizada por un competitivo precio determinado. Teniendo en cuenta la diversidad cultural y la diferencia regional de Brasil, muchas experiencias se han puesto en práctica, aisladamente, intentando incrementar y diversificar productos turísticos de calidad.

Sin embargo, dadas las necesidades, perfil y motivación de quienes buscan bienes y servicios turísticos, unas regiones se tornan más o menos atractivas que otras en términos de turismo debido a sus atributos endógenos y exógenos, es decir, internos y externos (Brasil, 2010). De ahí pues que las empresas y agencias de turismo necesitan trabajar la fidelización y la calidad del destino junto con propuestas vivenciales vinculadas a la prestación de servicios (Beni, 2004). Una mirada hacia los resultados del PRT revela que el desarrollo de la actividad turística es desigual tanto en relación al interior como en el litoral del país. Para Fonseca y otros (2019, p. 17), la categorización turística creada para estimular la competitividad entre los municipios ha venido reforzando la desigualdad espacial y conduciendo a la generación de indicadores referentes al desarrollo de la actividad turística que, además de dispares, mantienen las desigualdades tradicionales y promueven un desarrollo turístico desigual.

Por otra parte, también se identificaron diferencias en el aumento de las ofertas turísticas. Uno de los objetivos del PRT es internalizar el turismo brasileño; sin embargo, ha habido muchas dificultades para extender la actividad hasta más áreas del interior (Fonseca y otros, 2019, p. 164). Pasada una década y media desde la implantación de esta política pública, parece que la expansión del turismo aún es incipiente en el interior del país. Aunque existe un mayor interés gubernamental y compromiso político institucional, que ha llevado a la inserción del PRT en todo el país, aún no ha tenido los efectos deseados en el desarrollo de productos turísticos, ni ha conducido a un desarrollo satisfactorio del turismo en Brasil (Silva, 2020, p. 17). La falta de armonía entre el MTur y los grupos de gestión de las regiones turísticas está comprometiendo seriamente el éxito del PRT. Silva y Fonseca (2017) señalan que la interiorización de la actividad turística ha avanzado más en algunos estados que en otros, pero dichas acciones tienen una motivación política que, muchas de las veces, se sobrepone a decisiones racionales de contenido técnico, colaborativo y estructurante. Según dichos autores, existe una brecha significativa entre los supuestos propugnados en los planes nacionales y su materialidad en los municipios integrados en regiones turísticas.

Si bien el turismo es un factor de desarrollo local y sostenible, la gran dificultad del PRT para promover los productos turísticos a nivel regional y local consiste en que no logra promover una cooperación e integración efectiva entre los agentes involucrados en la actividad turística, requisitos considerados premisas básicas para su funcionamiento en la práctica (Trentin y 
Fratucci, 2013). Así, el turismo, como actividad, por ser un sector transversal y complejo, tiene todo el potencial para capitalizar las relaciones de ocio, recreación y entretenimiento, impulsando segmentos económicos y promoviendo la generación de ingresos directos e indirectos a todos los actores involucrados en esta actividad (Williams, 2004; Krippendorf's, 2009).

\section{Conclusión}

Considerada una de las áreas que menos han sufrido impacto con las recientes crisis económicas, el turismo puede ser desarrollado en diversos tipos de negocios, aunque la crisis social causada por el virus Sars-CoV-2 ha producido un efecto devastador en la actividad turística a nivel mundial. En este estudio se destacó la actividad del turismo desde la perspectiva del desarrollo sostenible, sin tener en cuenta los impactos causados por la enfermedad por coronavirus (Covid-19), y cómo dicho modelo podría contribuir al desarrollo socioeconómico, con la reducción de las desigualdades sociales regionales en Brasil.

Al investigar el Programa de Regionalización del Turismo (PRT) propuesto por el Ministerio de Turismo (MTur) se ha verificado su papel como propuesta brasileña de presentar un documento orientador del desarrollo turístico sostenible, constituyéndose en una muestra de valor utilitario para la gestión descentralizada y armónica de la actividad turística en los destinos de formación, conciliando los intereses económicos y los impactos sociales adversos de las localidades con la estrategia de desarrollo sostenible de una región o territorio.

El análisis de los datos presentados permite constatar la efectividad de la política de regionalización del turismo en Brasil por la capacidad de desarrollo e integración de municipios en la formación de regiones turísticas. Sin embargo, hay que señalar que la política de internacionalización promovida por el MTur a través del PRT aún no viene contribuyendo satisfactoriamente para impulsar el proceso de interiorización de la actividad turística en Brasil. No es evidente, en la práctica, la contribución del PRT en el sentido de establecer valores y prácticas que fortalezcan la gobernanza descentralizada de la actividad turística en Brasil. Por lo tanto, aún no es posible evaluar si este programa realmente produce cambios institucionales significativos que profundicen procesos verdaderamente regionalizados, con estructuras y mecanismos más descentralizados, participativos y también legítimos, además de ser más efectivos en estructurar políticas dirigidas al turismo, apoyados en la cooperación y participación de los distintos actores.

Hay señales de falta de armonía entre el MTur y los grupos de gestión de las regiones turísticas, hecho que compromete seriamente el éxito del PRT, y de que imperan las decisiones políticas por encima de aspectos técnicos pertinentes al desarrollo regional del turismo. Para la efectividad de este nuevo modelo de gobernanza de la actividad turística brasileña, es importante tener un rol protagónico de las organizaciones públicas y privadas que se inserten en los estados y municipios y la capacidad de acción que tienen. Según Endres y Pakman (2019: 5), se refiere a una capacidad que está sujeta al posicionamiento de estos actores en la red de relaciones establecida por la estructura de gobierno adoptada para la implementación del PRT en cada localidad. En esta perspectiva, es imperativo fortalecer la capacidad de gestión de los actores involucrados en el turismo y las relaciones institucionales que se establecen entre ellos para hacer más eficientes las acciones propuestas.

El resultado apunta al turismo como una actividad con gran potencial de desarrollar regiones brasileñas, pero exige que sean consideradas las especificidades de las mismas. En lo que se refiere a la sostenibilidad, la atención también debe ser dada en el sentido de considerar los aspectos sociales, culturales, económicos y ambientales en cada destino turístico.

Este estudio intentó contribuir a la literatura discutiendo la actividad turística como inductora del desarrollo socioeconómico y sostenible y sus resultados pueden servir de apoyo a estudios adicionales sobre el tema. Como sugerencia para el trabajo futuro, ante la relevancia del turismo y la necesidad de verificación de su efectividad a partir de políticas públicas, se ha identificado la necesidad de desarrollar investigaciones dirigidas a la construcción regional del turismo incluyendo a todos los actores involucrados en esta actividad, de modo a proporcionar condiciones de potenciar el desarrollo 
sostenible y reducir los impactos negativos generados por la economía turística.

\section{Referencias}

Ablas, L. (1991). Efeitos do turismo no desenvolvimento regional. Revista Turismo em Análise, 2(1), 42-52. https://doi.org/10.11606/issn.1984-4867.v2i1p42-52

Adams, C., Hoque, Z., \& McNicholas, P. (2006). Case studies and actions research. In Z. Hoque (org.), Methodological issues in accounting research: Theories and methods (pp. 361-373). London: Spiramus Press.

Arteaga Basurto, C., \& Solís San Vicente, S. [coords.]) (2001). La política social en la transición. Segunda Edición México: Universidad Nacional Autónoma de México, Escuela Nacional de Trabajo Social, pp. 356-371; 402-426.

Beni, M. C. (2004). Turismo: da economia de serviços à economia da experiência. Turismo, Visão e Ação, 6(3), 295-306. http://www.spell.org.br/documentos/ver/22082/turismo--da-economia-de-servicos-a-economia-da-experiencia/i/pt-br

Berry, A., \& Otley, D. (2004). Case-Based Research in Accounting. In Christopher Humphrey \& Bill Lee, The Real Life Guide to Accounting Research: A Behind the Scenes View of Using Qualitative Research Methods, Chapter 14. London: Elsevier, pp. 231-256.

Bowen, G. A. (2009). Document Analysis as a Qualitative Research Method. Qualitative Research Journal, 9(2), 27-40. 10.3316/QRJ0902027

Brasil (1988). Constituição da República Federativa do Brasil de 1988. Brasília: Diário Oficial da União (DOU) 05/10/1988, pp. 1. hhttp://www.planalto.gov.br/ccivil_03/constituicao/constituicao.htm

Brasil. (2019c). Decreto $n^{\circ}$ 9.791, de 14 de maio de 2019. Aprova o Plano Nacional de Turismo 2018-2022. http://www.planalto.gov.br/ccivil_03/_ato20192022/2019/decreto/D9791.htm.

Brasil. Ministério do Turismo (s.d.). Mapa do Turismo (2013-2019). Brasília: MTur. http://www.mapa.turismo.gov.br/mapa/init.html\#/home.

Brasil. Ministério do Turismo (2003). Plano Nacional do Turismo. Diretrizes, metas e programas. 2003-2007. Brasília: MTur. www.embratur.gov.br/0catalogo-documentos/Anuario/Plano_Nacional_do_Turismo.pdf

Brasil. Ministério do Turismo (2004a). Programa de Regionalização do Turismo - Roteiros do Brasil. Diretrizes Operacionais. Brasília: MTur.

Brasil. Ministério do Turismo (2004b). Programa de Regionalização do Turismo - Roteiros do Brasil. Diretrizes Políticas”. Brasília: MTur.

Brasil. Ministério do Turismo (2005). Programa de Regionalização do Turismo - Roteiros do Brasil: Módulo Operacional 7 - Roteirização Turística. Brasília: MTur.

Brasil. Ministério do Turismo (2007). Plano Nacional do Turismo: uma viagem de inclusão 2007-2010. Brasília: MTur.

Brasil. Ministério do Turismo (2010). Segmentação do turismo e o mercado. Brasília: MTur https://pt.slideshare.net/turismosantamaria/segmentao-do-turismoe-o-mercado

Brasil. Ministério do Turismo MTur. (2013a). Programa de Regionalização do Turismo: Diretrizes. Brasília: Brasília: MTur.

Brasil. Ministério do Turismo (2013b). Plano Nacional de Turismo (2013-2016): o turismo fazendo muito mais pelo Brasil" Brasília: MTur. https://bibliotecadigital.seplan.planejamento.gov.br/handle/123456789/925

Brasil. Ministério do Turismo (2014). Método para a Categorização do Mapa do Turismo Brasileiro. Brasília: MTur.

Brasil. Ministério do Turismo (2015a). Avaliação do programa de regionalização do turismo - roteiros do Brasil. Brasília: MTur. http://www.turismo.gov.br/sites/default/turismo/o_ministerio/publicacoes/downloads_publicacoes/Livro_Regionalizaxo.pdf

Brasil. Ministério do Turismo (2015b). Portaria $n^{\circ}$ 144, de 27 de agosto de 2015. Brasília: MTur. http://www.turismo.gov.br/legislacao/?p=822

Brasil. Ministério do Turismo (2016). Categorização dos Municípios das Regiões Turísticas do Mapa do Turismo Brasileiro. Brasília: Mtur, 2016. http://www.planalto.gov.br/ccivil_03/_ato2007-2010/2008/lei/111771.htm

Brasil. Ministério do Turismo (2017). Portaria $n^{\circ}$ 197, de 14 de setembro de 2017. Brasília: MTur. http://www.turismo.gov.br/assuntos/8198-portarian\%C2\%BA-197,-de-14-de-setembro-de-2017.html

Brasil. Ministério do Turismo (2018). Plano Nacional de Turismo - 2018-2022: Mais emprego e renda para o Brasil. Brasília: MTur. http://www.turismo.gov.br/images/mtur-pnt-web2.pdf

Brasil. Ministério do Turismo (2019a). Portaria $n^{\circ}$ 271, de 23 de agosto de 2019”. Brasília: MTur, Publicado en 26/08/2019, Edición 164, Sección: 1, p. 78 https://www.in.gov.br/en/web/dou/-/portaria-n-271-de-23-de-agosto-de-2019-212423289.

Brasil. Ministério do Turismo (2019b). Regionalização: sensibilização e mobilização. Brasília: MTur. http://www.regionalizacao.turismo.gov.br/images/mturcartilha-promocional-final.pdf

Brasil. Ministério do Turismo (2019d). Mapa do Turismo Brasileiro. Brasília: MTur. http://www.regionalizacao.turismo.gov.br/images/conteudo/LIVRO_Mapa.pdf

Carvalho, L.O.R., Duarte, F. R., Menezes, A. H. N., \& Souza, T. E. S. (2019). Metodologia científica: teoria e aplicação na educação à distância. Petrolina-PE. 
Research, Society and Development, v. 10, n. 11, e05101119153, 2021

(CC BY 4.0) | ISSN 2525-3409 | DOI: http://dx.doi.org/10.33448/rsd-v10i11.19153

Cerdá Tena, E., \& Khalilova, A. (2016). Economía circular. Revista Economía Industrial, 401, 11-20. https://www.mincotur.gob.es/Publicaciones/Publicacionesperiodicas/EconomiaIndustrial/RevistaEconomiaIndustrial/401/CERD\%C3\%81\%20y\%20KHALILOVA.pdf

CNM - Confederação Nacional de Municípios (2012). CNM Turismo: o Turismo como gerador de desenvolvimento. Brasília/DF: CNM [Coletânea Gestão Pública Municipal - Gestão 2013-2016] Vol. 11, 56 p.

Cota Elizalde, B.D. (2011). Política social y ciudadanía; familias jornaleras agrícolas asentadas en Sinaloa: Estudio de caso. In: Gil Rodríguez, Reyna (Coord.), Retos de la investigación para impulsar el desarrollo humano y social. $1^{\mathrm{a}}$. Edición [Memorias del XV Encuentro Nacional y V Internacional de Investigación en Trabajo Social], Chilpancingo, Gro., México: Universidad Sentimientos de la Nación A.C., pp. 135-147, diciembre. Retrieved from http://www.ts.ucr.ac.cr/binarios/congresos/nac/mx/mx-con-eniits-015-01.pdf

Cruz Moreno, I.A., \& Zizumbo Villarreal, L. (2017). Alcances de la política social y el turismo rural para el desarrollo local en San Mateo Almomoloa México. Estudios y Perspectivas en Turismo, 26, 944- 963. http://hdl.handle.net/20.500.11799/78993

Dalonso, Y. S., Lourenço, J. M., Remoaldo, P. C., \& Panosso Netto, A. (2012) . Public policies for tourism in Brazil: An analysis of the National Tourism Plan for cities. Configurações, 10, 185-198.

Embratur - Agência Brasileira de Promoção Internacional do Turismo (1998a). Diretrizes do Programa Nacional de Municipalização do Turismo. $3^{\mathrm{a}}$ versão. Brasília: EMBRATUR.

Embratur - Agência Brasileira de Promoção Internacional do Turismo (1998b). Procedimentos do Programa Nacional de Municipalização do Turismo. Brasília: EMBRATUR.

Embratur - Agência Brasileira de Promoção Internacional do Turismo (1999). Diretrizes do Programa Nacional de Municipalização do Turismo. Brasília: EMBRATUR.

Embratur - Agência Brasileira de Promoção Internacional do Turismo (2002). Retratos de uma caminhada: PNMT 8 anos. Brasília: EMBRATUR.

Endres, A. V., \& Pakman, E. T. (2019). A governança das políticas de turismo: o papel dos espaços de participação na perspectiva da análise de redes e da teoria institucional. RBTUR - Revista Brasileira de Pesquisa em Turismo, 13(1), 1-18. https://doi.org/10.7784/rbtur.v13i1.1431

Fonseca, M. A. P., Bicalho, D. M., Sousa, R. M., \& Silva, R. C. (2019). Programa de Regionalização do Turismo: Análise comparativa dos indicadores turísticos nos estados brasileiros de Santa Catarina, Rio de Janeiro e Rio Grande do Norte. Ar@cne. Revista Electrónica de Recursos de Internet sobre Geografía y Ciencias Sociales, 234, 1-20.

Frey, B. B. (2018). Document Analysis. In Bruce B. Frey (ed.), The SAGE Encyclopedia of Educational Research, Measurement, and Evaluation. Thousand Oaks, CA: SAGE Publications, Inc. https://dx.doi.org/10.4135/9781506326139

García, A. C. (1993). Análisis documental: el análisis formal. Revista General de Información y Documentación, 3(1), 11-19.

Gonsalves, E. P. (2003). Iniciação à pesquisa científica. 3. ed. Campinas, SP: Alínea.

González Herrera, M. (2007). Políticas turísticas para el desarrollo sostenible del turismo cultural / San Juan de los Remedios, Cuba. Teoría y Praxis, 3, 53-63.

González Herrera, M. (2006). Gestión preventiva de impactos ambientales - Implantación geoespacial del turismo en el sector oeste de Cayo Santa María, Jardines del Rey - Cuba. Estudios y Perspectivas en Turismo, 15, 350-366.

Ignarra, L.R. (1991). Fundamentos do Turismo. São Paulo: Pioneira.

Köche, J. C. (2007). Fundamentos de metodologia científica: Teoria da ciência e iniciação cientifica. 24. ed. Petrópolis: Editora Vozes.

Krippendorf’s, J. (2009). Sociologia do Turismo: para uma nova compreensão do lazer e das viagens. São Paulo: Aleph.

Massaro, M., Dumay, J., \& Guthrie, J. (2016). On the shoulders of giants: undertaking a structured literature review in accounting. Accounting, Auditing \& Accountability Journal, 29(5), 767-801. https://doi.org/10.1108/AAAJ-01-2015-1939

Monteiro Neto, A.; Castro, C. N.; \& Brandão, C. A. (2017). Desenvolvimento territorial no Brasil: reflexões sobre políticas e instrumentos no período recente e propostas de aperfeiçoamento. In: Monteiro Neto, A.; Castro, C. N.; \& Brandão, C. A. (Orgs.) Desenvolvimento regional no Brasil: políticas, estratégias e perspectivas, Rio de Janeiro: IPEA, pp. 37-65.

OIT - Organización Internacional del Trabajo (2010). Cambios y desafíos en el sector de la hotelería y el turismo. Ginebra: OIT. https://www.ilo.org/wcmsp5/groups/public/---ed_dialogue/---sector/documents/meetingdocument/wcms_162207.pdf

OIT - Organización Internacional del Trabajo (2011). Guía Práctica sobre la Reducción de la Pobreza a través del Turismo. Ginebra: OIT. https://www.ilo.org/wcmsp5/groups/public/---ed_dialogue/---sector/documents/instructionalmaterial/wcms_171733.pdf

OMT - Organização Mundial do Turismo (2018). Panorama OMT del turismo internacional. [Edición 2018] Madrid: OMT. https://www.eunwto.org/doi/pdf/10.18111/9789284419890

OMT - Organização Mundial do Turismo (2019). Panorama OMT del turismo internacional. [Edición 2019] Madrid: OMT. https://www.eunwto.org/doi/pdf/10.18111/9789284421237

OMT - Organização Mundial do Turismo (2020). Panorama OMT del turismo internacional. [Edición 2020] Madrid: OMT. https://www.eunwto.org/doi/epdf/10.18111/9789284422746

Pereira, A. S., Shitsuka, D. M., Parreira, F. J., \& Shitsuka, R. (2018). Metodologia da pesquisa científica. Santa Maria, RS: UFSM, NTE. 
Research, Society and Development, v. 10, n. 11, e05101119153, 2021

(CC BY 4.0) | ISSN 2525-3409 | DOI: http://dx.doi.org/10.33448/rsd-v10i11.19153

Ryan, B., Scapens, R. W., \& Theobald, M. (2002). Research Method and Methodology in Finance and Accounting. London: Academic Press, Thomson Business Press.

Sansolo, D.G., \& Cruz, R.C.A. (2003). Plano Nacional de Turismo: uma análise crítica. Caderno Virtual de Turismo, 3(4), 1-6.

Scapens, R. (2004). Doing Case Study Research. In Christopher Humphrey \& Bill Lee (eds.). The Real Life Guide to Accounting Research: A Behind the Scenes View of Using Qualitative Research Methods. London: Elsevier, Chapter 15, pp. 257-280.

Sebrae - Serviço Brasileiro de Apoio às Micro e Pequenas Empresas (2016). Cenários Prospectivos. O Turismo Brasileiro de 2016 A 2018. Rio de Janeiro: SEBRAE. http://www.bibliotecas.sebrae.com.br/chronus/ARQUIVOS_CHRONUS/bds/bds.nsf/3d98ea3e4e49602196a85fd6951faac1/\$File/7489.pdf

Silva, L. R. M., \& Andrade, J. R. L. (2008). Programa de regionalização do turismo e sua aplicação em comunidades autóctones: o caso de Poço Redondo e Canindé do São Francisco - SE. Caderno Virtual de Turismo, 8(2), 15-22.

Silva R. C., \& Fonseca, M.A.P. (2017) Os investimentos do Ministério do Turismo e o Programa de Regionalização do Turismo: desencontros da política governamental no interior potiguar. Caderno Virtual de Turismo 17(3), 148-165. https://www.redalyc.org/articulo.oa?id=115454650010.

Silva, T.D.P., \& Nogueira, C.R.D. (2020). Política pública de turismo como fator de promoção do desenvolvimento local e regional. In Pereira, A., Seabra, G.F., \& Nogueira, C. R.D. (Orgs). Desafios e dinâmicas espaciais do turismo receptivo. Ituitaba, MG: Editora Barlavento.

Steffen, W. et al. (2015). Planetary boundaries: Guiding human development on a changing planet. Science, 347(6223), 1-10. https://science.sciencemag.org/lookup/doi/10.1126/science.1259855

Trentin, F., \& Fratucci, A. C. (2013). National Policy of Tourism in Brazil: From Municipalisation to Regionalization. China-USA Business Review, 12(7), 718-727.

Tripoli, S., \& Bender, K. (2010). Descriptive Studies. In Thyer, B. (Org.). The handbook of social work research methods. 2 ed. California: Sage Publications, Inc.

UNWTO - United Nations and World Tourism Organization (2013). Sustainable Tourism for Development Guidebook (2013). Madrid (Spain): UNWTO https://www.e-unwto.org/doi/pdf/10.18111/9789284415496

Vargas, R.M. (2018). El método de construcción regional del turismo en México. Una aproximación espacial. Rosa dos Ventos - Turismo e Hospitalidade, 10(4), 637-652.

Vieira, A.R.M. (2015). Planejamento e políticas públicas de turismo: análise dos modos operacionais do programa de regionalização do turismo em São Luis-MA. [Dissertação de Mestrado Profissional em Turismo]. Brasília: Universidade de Brasília.

Williams, A. M. (2007). Para uma Economia Política do Turismo. In Alan A. Lew, C. Michael Hall, \& Allan M. Willians, Compêndio de turismo [trad. Luís Couceiro Feio et al.]. Lisboa: Instituto Piaget. 